\title{
PGF isoforms, PLGF-1 and PGF-2 and the PGF receptor, neuropilin, in human breast cancer: Prognostic significance
}

\author{
ASTRID ESCUDERO-ESPARZA ${ }^{1}$, TRACEY A. MARTIN ${ }^{1}$, \\ ANTHONY DOUGLAS-JONES ${ }^{2}$, ROBERT E. MANSEL ${ }^{1}$ and WEN G. JIANG ${ }^{1}$ \\ ${ }^{1}$ Metastasis and Angiogenesis Research Group, University Department of Surgery and \\ ${ }^{2}$ Department of Pathology, Cardiff School of Medicine, Cardiff University, Cardiff CF14 4XN, UK
}

Received August 6, 2009; Accepted October 5, 2009

DOI: $10.3892 /$ or_00000667

\begin{abstract}
Placenta growth factor (PLGF) is a member of the vascular endothelial growth factor (VEGF) family, a group of angiogenic growth factors. Recently, isoforms have been identified. This study examined PLGF-1, PGF-2 and its receptor neuropilin-1 levels in human breast cancer in relation to patient's clinical parameters and how changes in expression may be linked to prognosis of the disease. PLGF-1, PGF-2 and neuropilin-1 transcript expression and distribution were examined quantitatively using real-time quantitative polymerase chain reaction (Q-PCR) on a cohort of human breast cancer $(n=114)$ and background breast tissue $(n=30)$ with a 10-year follow-up. Protein expression was assessed by an immunohistochemical method. We demonstrate that PLGF-1 transcript levels were significantly elevated when comparing tumours from patients with poor outcome and patients who remained disease-free $(\mathrm{P}=0.03)$, indicating a potential prognostic value. Immunohistochemistry demonstrated a marked increased in PGF-2 expression in tumour section compared with normal tissues $(\mathrm{P}<0.05)$. PGF-2 transcripts, showed little change in expression between tumour and background. High levels of PLGF-1 and PGF-2 were seen in ERß-negative breast tumour tissues. Neuropilin transcript was below detection in substantial portion of the samples and was more frequently detected in high grade tumours $(\mathrm{P}=0.008$ vs. low grade) and in tumours from patients who died of breast cancer $(\mathrm{P}<0.001$ vs. those who remained disease-free). Our study shows that PLGF isoforms PLGF-1 and PGF-2 and
\end{abstract}

Correspondence to: Dr Astrid Escudero-Esparza, Metastasis and Angiogenesis Research Group, Department of Surgery, Cardiff School of Medicine, Cardiff University, Cardiff CF14 4XN, UK E-mail: escudero-esparzaa@cardiff.ac.uk

Key words: breast cancer, immunohistochemistry, neuropilin-1 receptor, placenta growth factor, prognosis, real-time quantitative polymerase chain reaction, vascular endothelial growth factor indeed their receptor neuopilin, have an aberrant pattern of expression and that high levels of the PLGF-1 and neuropilin are linked to a poor prognosis.

\section{Introduction}

Angiogenesis, the process involving growth of new blood vessels, is an essential component of the metastatic pathway. These vessels provide the principal route by which tumour cells exit the primary tumour site and enter the circulation, without which the tumour would fail to develop. Tumour angiogenesis is regulated by pro-angiogenic factors [including FGF (fibroblast growth factor) and VEGF (vascular endothelial growth factor)] and anti-angiogenic factors such as thrombospondin-1 (TSP-1), endostastin and angiostastin (1). Among the growth factors implicated in tumour angiogenesis, VEGF appears to be a major contributor in angiogenesis in solid tumours. Different VEGF family member appears to have a specific function: VEGF-C and VEGF-D both are primarily lymphangiogenic factors, while VEGF and VEGF-B are primarily angiogenic (2). In addition, VEGF directly promotes proliferation and/or migration of VEGF receptor-expressing tumour cells and they are also involved in mobilization of endothelial and haematopoietic stem cells (HSCs) from the bone marrow and their recruitment at the tumour site where they contribute to tumour angiogenesis (3).

Placenta growth factor, PGF, is a member of the VEGF family which is mainly expressed in the placenta and within the placenta the major cellular source is trophoblast (4). It is also found expressed in vascular endothelium, umbilical vein endothelial cells, the eye, uterine natural killer cells and dentine matrix. It is a homodimeric glyprotein that displays homology with VEGF, sharing 53\% similarity in its overall amino acid residues. Human PGF gene has been mapped to chromosome 14q24. PGF-coding sequence is encoded by seven exons spanning an 800-kb long DNA interval (5). As a result of alternative splicing the primary PGF transcript, PGF has at least three isoforms, PLGF-1, PGF-2, also known as PLGF-2 (4-9) and PGF-3, also known as PLGF-3 $(5,8)$. However, only one study has described a fourth isoform, PLGF-4 in human trophoblast and endothelial cells (4). 
PGFs mediate their effects through VEGFR-1. PGF-2 has a 21-amino acid heparin binding domain and is also able to bind both of the known PGF receptors: neuropilin-1 (Nrp-1) and FLT-1 while PLGF-1 and PGF-3, both lack the heparin binding domain, and bind only FLT-1.

Nrp-1 is a $120-130 \mathrm{kDa}$ membrane glycoprotein, first identified as a neuronal receptor for specific secreted members of semaphorin family involved in axon repulsion. The extracellular region consists of three domains: the a1/a2 domain, which is homologous to the complement-binding motifs; the b1/b2 domain, which is homologous to coagulation factors $\mathrm{V}$ and VIII; and the c domain, which is homologous to meprin, A5 and receptor tyrosine phosphatise $\mu$ and is designated MAM (10).

Several studies on VEGF and PGF have revealed that they exert similar biological functions in stimulating the growth of vascular endothelial cells (6). PGF promotes motility and invasiveness, two main features associated with the epithelial to mesenchymal transformation that characterizes metastasis (9). In vitro results available on the angiogenesis role of PGF are inconsistent, some studies have revealed that PGF binding to VEGFR-1 (FLT-1) did not produce any growth or angiogenesis in endothelial cells (ECs), while others show that PGF/VEGFR-1 signalling promotes EC viability and angiogenesis. It seems clear thought that in placenta, PGF has a direct effect on endothelial cells, by enhancing its own signalling and by amplifying VEGF-driven angiogenesis (5).

Breast cancer is the most common form of malignancies in females in the UK and metastastasis of breast cancer is common. About $7 \%$ of patients with breast cancer present with widespread metastases at the initial presentation. The most common sites of metastasis are bone, lungs, liver, chest and the central nervous system. The role of VEGF in breast cancer has been well documented. However, the role of PGF isoforms and the PGF receptor neuropilin is not established. We have previously reported that transcript levels of PLGF are raised in aggressive breast tumours (11). To clarify the role of PLGF-1 and PGF-2 and its receptor, neuropilin, in breast cancer and its prognostic significance, we examined the expression of both isoforms and how changes in expression may be linked to prognosis of the disease, through analyses of the transcripts and proteins of these molecules, in a cohort of human breast cancer specimens.

\section{Materials and methods}

Human breast specimens. A total of 144 breast samples were obtained from breast cancer patients (30 background normal breast tissue and 114 breast cancer tissue), with consent of the patients and the Ethics Committee. These tissues were collected immediately after mastectomies and snap-frozen in liquid nitrogen. The pathologist (A.D.J.) verified normal background and cancer specimens and it was confirmed that the background samples were free from tumour deposit.

Polymerase chain reaction (PCR). Human breast cancer tissue was screened for endogenous expression of PLGF-1 and PGF-2.

Conventional PCR was performed using cDNA together with PCR master mix using respective primers. PLGF-1 and
PGF-2 forward and reverse primers were 5'-ATGCCGGTCA TGAGGCTGTTCCCTTGCTT-3' and 5'-TTACCTCCGGG GAACAGCATCGCCGCAC-3', two products being distinguished by their charactistic size differences. The reaction conditions were: $94^{\circ} \mathrm{C}$ for $5 \mathrm{~min}, 94^{\circ} \mathrm{C}$ for $15 \mathrm{sec}, 61^{\circ} \mathrm{C}$ for $30 \mathrm{sec}, 72^{\circ} \mathrm{C}$ for $1 \mathrm{~min}$ and the final extension phase at $72^{\circ} \mathrm{C}$ for $10 \mathrm{~min}$ for 42 cycles. The PCR products were separated on a $1.4 \%$ agarose gel and electrophoretically separated. The gel was then stained with ethidium bromide prior to examination under ultraviolet light, and photographs were taken.

Real-time quantitative polymerase chain reaction ( $Q-P C R)$. The Q-PCR used was the Amplifluor ${ }^{\mathrm{TM}}$ Uniprimer $^{\mathrm{TM}}$ system (Intergen Company Oxford, UK) and the Thermo-Start ${ }^{\circledR}$ (ABgene, Epsom, Surrey, UK), as previously reported $(12,13)$. Specific primers were designed by the authors using Beacon Designers software (Biosoft, Palo Alto, CA, USA) and manufacturated by Invitrogen (Invitrogen Life Technologies, Paisley, UK). Using the Icycler IQ system (Bio-Rad, Hemel Hempstead, UK), which incorporates a gradient thermocycler and a 96-channel optical unit, the breast samples cDNA were simultaneously assayed with a purified plasmids as internal standard. The level of each molecule cDNA (copies/50 ng RNA) in the breast samples was calculated. Q-PCR for B-actin was also performed on the same samples, to correct for any residual differences in the initial level of RNA in the specimens (in addition to spectrophotometry). Primer pairs for Q-PCR were as follows: PLGF-1 forward and reverse primers were 5'-GTTCTCTCA GCACGTTCG-3' and 5'-ACTGAACCTGACCGTACACAT CGCCGCACCTTTC-3' and PGF-2 forward and reverse primers were 5'-GGAGCTGACGTTCTCTCAG-3' and 5'-ACT GAACCTGACCGTACAGTTACCTCCGGGGAACAG-3'. Primers for neuropilin-1 forward and reverse were 5'-TCAA CTTCAACCCTCACTTC-3' and 5'-AACTTTCCCCTAAA ATGTCC-3'.

Immunohistochemical staining of breast specimens and densitometry analysis. This was based on methods we previously described (14-16). Frozen sections of breast tumour and back-ground tissue were cut at a thickness of $6 \mu \mathrm{m}$ using a cryostat (Leica). The sections were mounted on super frost microscope slides, air-dried and then fixed in a mixture of $50 \%$ acetone and $50 \%$ methanol. The sections were then placed in Optimax wash buffer (Menerium, Oxford, UK) for 5-10 min to rehydrate. Sections were incubated for $20 \mathrm{~min}$ in a horse serum albumin blocking solution and probed with PLGF-1, PGF-2 and neuropilin-1 antibody (1:100 dilution), and without primary antibody as a negative control to verify the binding specificity. Primary antibodies were purchased from Santa Cruz Biotechnologies Inc. (Santa Cruz, CA, USA). Following extensive washings, sections were incubated with the secondary biotinylated antibody and biotinylation solution, separated by extensive washings. Diaminobenzine chromogen (ABC Standard kit, Vector Laboratories, Peterborough, UK) was then added to the sections and incubated in the dark for $5 \mathrm{~min}$. Sections were counterstained in Gill's haematoxylin and dehydrated in ascending grades of methanol before clearing in xylene and mounting under a cover slip. Semi-quantification of staining intensity 
was carried out using Optimas package as we previously described (13). Immunohistochemical photographs of normal and tumour sections $(n=20)$ were assessed as previously described (15).

Statistical analysis. Statistical analyses were carried out using paired samples Student's t-test (mean \pm SD), Fisher's test (two-tailed) and by the non-parametric Mann-Whitney test (IQR) where appropriate. A P-value $\leq 0.05$ was defined as statistically significant. Fisher's exact tests were used for analysing neuropilin, the transcript of which was below detection in a good proportion of the tissues. Statistic tests were performed using SPSS 10.0 software (SPSS Inc., Chicago, IL, USA).

\section{Results}

Expression of PLGF-1 and PGF-2 in breast cancer tissue. PLGF-1 and PGF-2 transcripts were detected in breast cancer tissue and were seen as two discrete products, 447 and 512 bp in size, respectively, Fig. 1.

Immunohistochemical staining of breast specimens. Breast cancer tissue stained positively for PLGF-1, PGF-2 and neuropilin-1. The PLGF-1 and PGF-2 protein level was dramatically elevated in the breast tumour specimens compared with the normal breast tissue (Fig. 2). Staining intensity of PLGF-1 and PGF-2 in tumour tissues were significantly higher than in the normal breast tissue (mean $\pm \mathrm{SD}$ $131 \pm 53.40$ vs. $103.105 \pm 22.09, \mathrm{P}=0.031949,172.82 \pm 40.33$ vs. $102.05 \pm 46.65 \mathrm{P}=0.000117$, for $\mathrm{PlGF}-1$ and $\mathrm{PGF}-2$, respectively.

In neuropilin-1, the pattern of staining correlates with the staining for PGF-2 in breast cancer tissue compared with normal breast tissue (Fig. 3).

Quantification of PLGF-1 and PGF-2 transcripts in human breast tissues. We quantified the PLGF-1 and PGF-2 transcripts in the breast specimens (tumour $n=114$, background $\mathrm{n}=30$ ) using real-time Q-PCR (all values are displayed as mean transcript copies). We show that the PLGF-1 expression value in normal breast tissue was $182 \pm 107$, compared with $77 \pm 24$ in the breast cancer tissue (Fig. 4A), however, this did not reach a level of significance $(\mathrm{P}=0.34)$.

PGF-2, expression value in cancer tissue was $1548 \pm 291$, compared with normal breast tissue $984 \pm 125$. Levels of PGF-2 were higher in cancer tissue, although this did not reach a level of significance $(\mathrm{P}=0.077)$.

PGF-2 was sequentially increased with increasing Nottingham Prognostic Index (NPI), (Fig. 4C), status from $940 \pm 89.7$ in NPI 1 (NPI <3.5) tumours to $2913 \pm 1699$ in NPI 3 tumours (NPI >5.4). PLGF-1 was increased in NPI 2 (NPI 3.5-5.4) tumours (124.2 \pm 76.4 vs. $52.5 \pm 13.6$ in NPI 1$)$ but showed a little change in NPI 3 tumours $(61.7 \pm 16.5)$. However, these changes did not reach significance $(\mathrm{P}=0.67)$.

When expression was compared with tumour grade, PLGF-1 showed no obvious correlation (grade 1, 192 \pm 143 ;

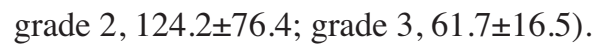

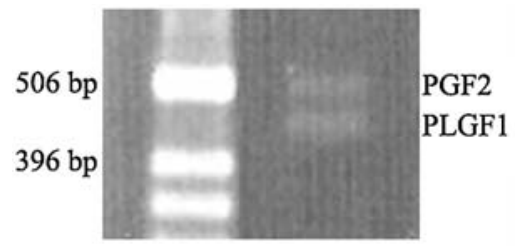

Figure 1. PCR analyses of PLGF-1 and PGF-2 isoform expresses by breast cancer tissue samples. A DNA ladder was included for size approximation. PLGF-1 is reported to be 447 base pair while PGF-2 is 512 base pair.

PGF-2 expression was increased in grade 3 when compared to grade 1 (grade $11773 \pm 623$ vs. $4545 \pm 3677$ ) although this did not reach significance $(\mathrm{P}=0.49)$.

PLGF-1 and PGF-2 and estrogen receptor status. No significant correlation was seen between PLGF-1, PGF-2 and ER status. PLGF-1 and PGF-2 expression appears to correlate with estrogen-receptor $\beta$ (ERß) grouping (ERßpositive $n=75$ and ERß-negative $n=22$ ). Levels of both PLGF-1 and PGF-2 were found to be significantly elevated in ERßnegative patients $(\mathrm{P}=0.014$ for $\mathrm{PLGF}-1$ and $\mathrm{P}=0.0004$ for PLGF-2) reaching statistical significance (Fig. 5).

PLGF-1 and PGF-2 and nodal status. PLGF-1 and PGF-2 in the node-positive tumours $(\mathrm{n}=47)$ were elevated compared with node-negative patients $(\mathrm{n}=54)(52.5 \pm 14$ vs. $104 \pm 52 ; \mathrm{P}=0.34$ for PLGF-1 and $941 \pm 90$ vs. $2231 \pm 632$; $\mathrm{P}=0.049$ for PGF-2), (Fig. 4B).

PLGF-1 and PGF-2 and survival status. We assessed the survival status of the breast cancer patient in association with PLGF-1 and PGF-2 levels, with a period of 10 years median. Patients were classified as 1) disease-free, 2) with metastatic disease, 3) with local recurrence of breast cancer, and 4) dead as a result of breast cancer.

Patients who had died of breast cancer had a significantly elevated levels of PLGF-1 ( $\mathrm{P}=0.03)$, (Fig. 6). However, the same was not seen with PGF-2 ( $\mathrm{P}=0.7857)$.

Expression of neuropilin-1 receptor. Neuropilin-1 transcript expression in the breast specimens was examined using realtime Q-PCR. There were a substantial number of samples in which neuropilin transcript was below detections. Samples were subsequently separated into two groups. Those where the neuropilin-1 receptor transcript was detectable (positive) and those where the receptor was below detection (negative). The difference between the neuropilin-1 receptor status and their correlation with a number of other important factors in breast cancer were analysed using a two-tailed Fisher's test and were regarded as significant at $\mathrm{P}<0.05$, Table I. A significantly higher portion of patients classified as having a poor prognosis (Nottingham Prognostic Index, NPI, >5.4) were found to be receptor-positive compared to those classified with a good NPI (<3.4) status $(\mathrm{P}=0.033)$.

When looking at tumour grade of differentiation, we found that those patients classified with a low grade tumour (Grade 1) were significantly more likely to be negative for the detection of neuropilin-1 when compared to patient samples with higher grade classification (Grade 3$)(\mathrm{P}=0.008)$. 

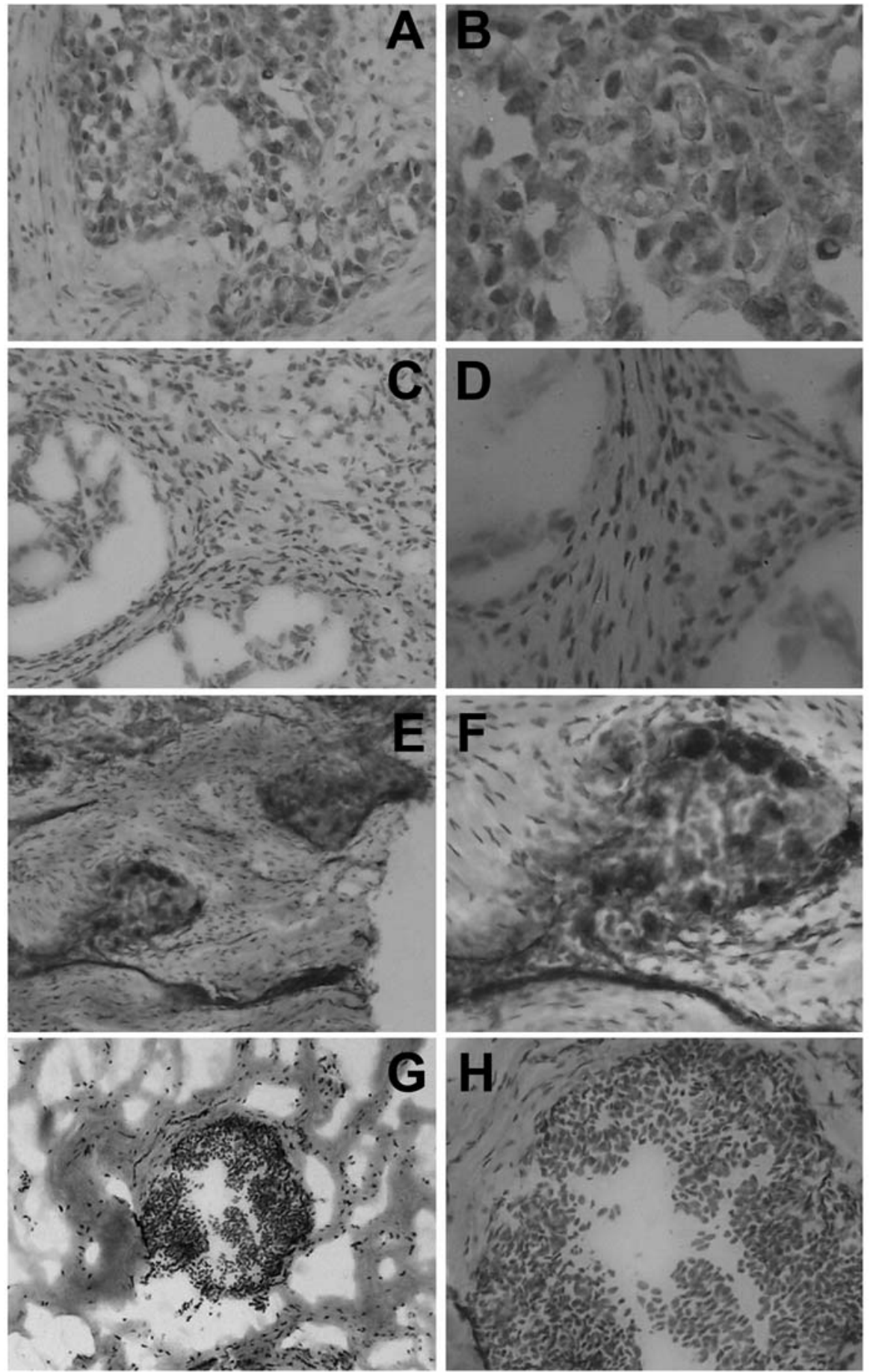

Figure 2. Immunohistochemical staining for PLGF-1 and PGF-2 of human breast specimens. (A and B) Strong cytoplasmic staining of PLGF-1 in human breast cancer tissue. (C and D) Negative staining in normal background breast tissue for PLGF-1. (E and F) Strong cytoplasmatic staining for PGF-2 in human cancer tissue, $(\mathrm{G}$ and $\mathrm{H}$ ) negative staining in normal background breast tissue for PGF-2. These sections demonstrate that both PLGF-1 and PGF-2 are expressed at far higher levels in the breast tumour specimens compared with the normal breast tissue (original magnification x100).

Similarly, samples obtained from patients with a good clinical outcome (disease-free) were found to be significantly more likely to be neuropilin-1-negative than those from patients who had died as a result of the disease $(\mathrm{P}<0.001)$.

A correlation between ERß status and neuropilin-1 status was also observed. Patients who were ERß-positive were also found to be mainly neuropilin-1-negative when compared to those patients who were $E R ß$-negative $(\mathrm{P}=0.042)$.

\section{Discussion}

PGF is a member of the VEGF family and is known to be a powerful angiogenic factor. VEGF has arisen as a pivotal regulator of the angiogenic process in physiological and pathological conditions as in various human malignancies, including breast cancer. Although PGF has been studied in a number of clinical tumour types, little is known about the different isoforms, PLGF-1, PGF-2, PGF-3 and their prognostic 

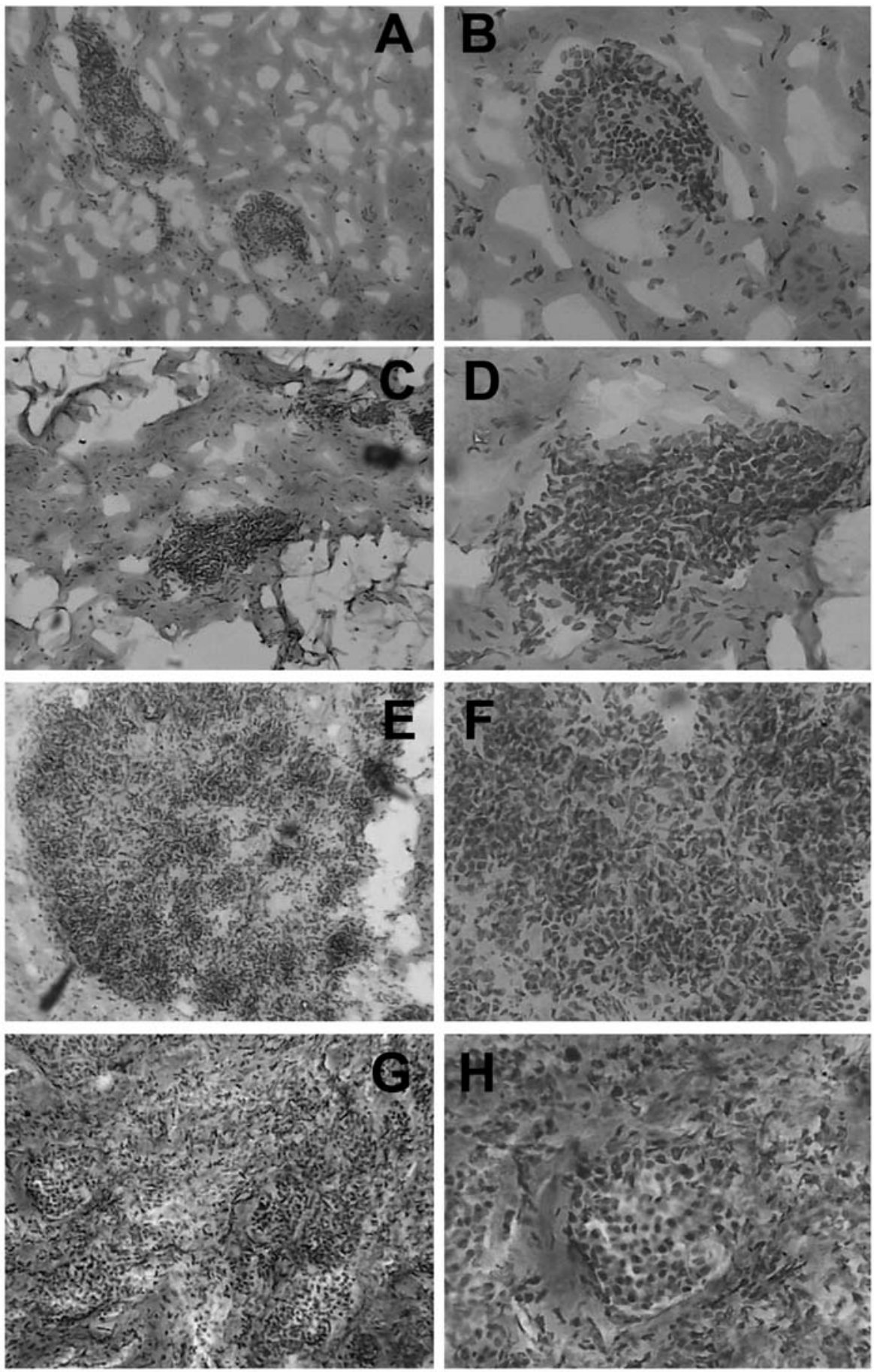

Figure 3. Immunohistochemical staining for neuropilin-1 of human breast specimens. (A-D) Negative staining in normal background breast tissue for neuropilin-1. (E-H) A good level of neuropilin-1 in human breast cancer tissue, the staining tends to be cytoplasmatic. The pattern of staining of neuropilin-1 correlates with the staining of PGF-2, which shows a markedly increased staining in human breast cancer tissue compared to normal background breast tissue.

significance in human breast cancer. The current study evaluated these factors in relation with PLGF-1 and PGF-2 and the PGF receptor neuropilin. We investigated the expression of PLGF-1 and PGF-2, at the protein level and the messenger RNA level and whether it has a significant value in the prognosis of the breast cancer patients. To the best of our knowledge this study is the first reporting that both isoforms were detected in all breast cancer tissues and the matched normal tissues, however, only limited reports have studied mRNA expression of PGF irrespective of the isoform.
High levels of PGF-2 mRNA were found in breast tumour tissues, this correlated with the IHC staining intensity, whereas PLGF-1 showed a higher expression in normal tissue although not statistically significant. This result does not correlate with the IHC for this isoform. This may be due to the nondiscriminative nature of Q-PCR. We cannot determine which cells exclusively express PLGF-1. In IHC, we can observe where the higher staining intensity is, relative to weak staining. Moreover, IHC is a semi-quantitative method. We confirm that PLGF-1 and PGF-2 mRNA levels were 

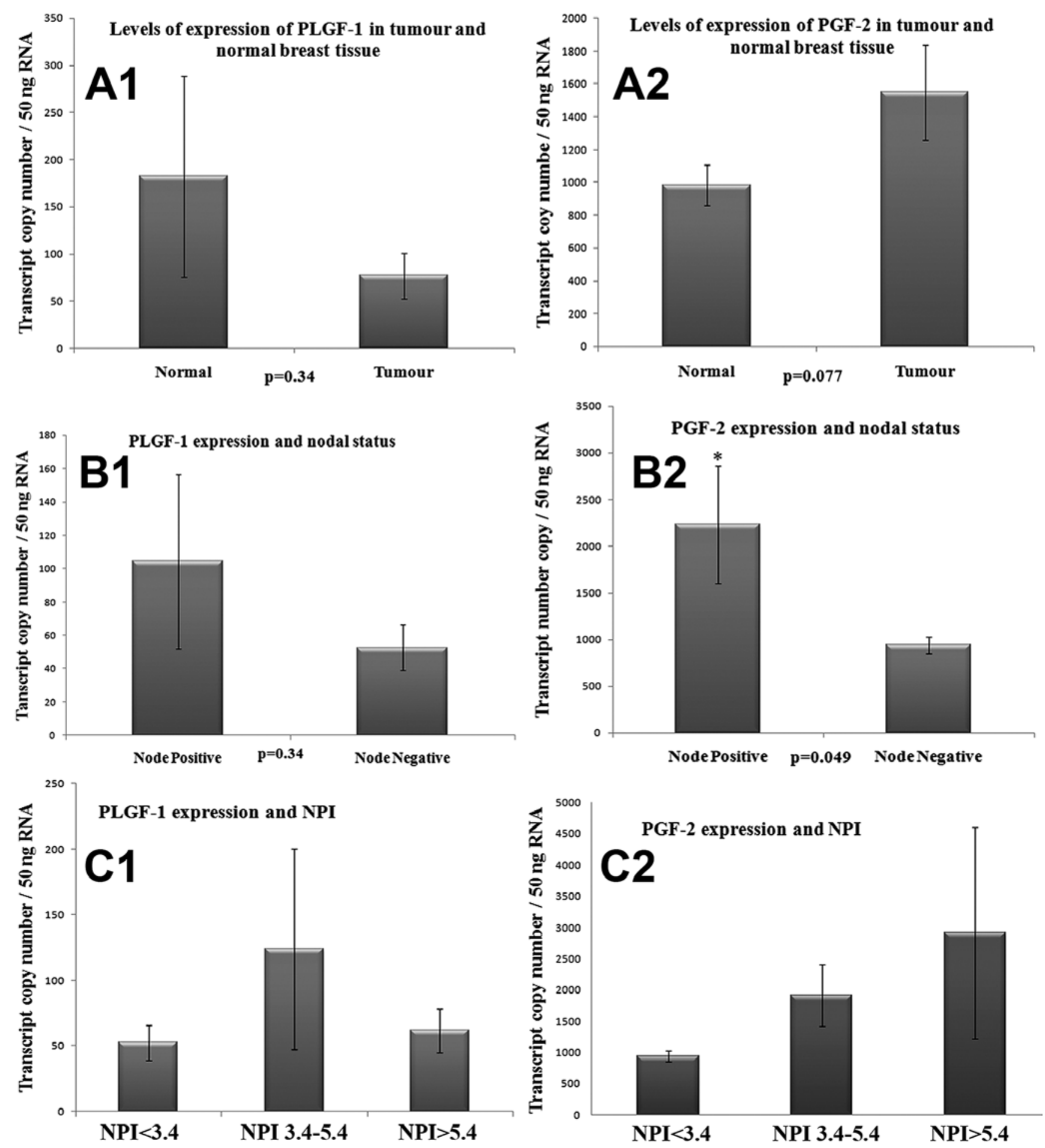

Figure 4. Levels of transcript of PLGF-1 and PGF-2. (A) PIGF1 (A1) and PGF-2 (A2) in tumour samples in comparison to background. (B) Levels of transcript of PLGF-1 (B1) and PGF-2 (B2) expression in node-positive tumours compared with node-negative tumours. (C) Levels of PLGF-1 (C1) and PGF-2 (C2) transcripts in correlation with the Nottingham Prognostic Index (NPI).

dramatically elevated when looking at clinical outcome for these patients. Levels of PLGF-1 and PGF-2 were higher in tumour tissues from patients who died from breast cancer compared with those who survived. In keeping with this trend, patients who died from breast cancer were also found to have a higher proportion of the receptor for PLGF-1 and PGF-2, neuropilin-1. This high degree of expression of PLGF-1 and PGF-2 together with the presence of neuropilin-1 was indicative for a poor prognosis for patient. Therefore, both isoforms demonstrate strong prognostic potential.

The Nottingham prognostic index (NPI), has been used as a prognostic indicator, with its reliability being validated in several studies (17). Both, PLGF-1 and PGF-2 showed a high expression at NPI-2 and NPI-3, correlating these results with the poor prognosis we described before. Similarly, the proportion of patients having positive readings 

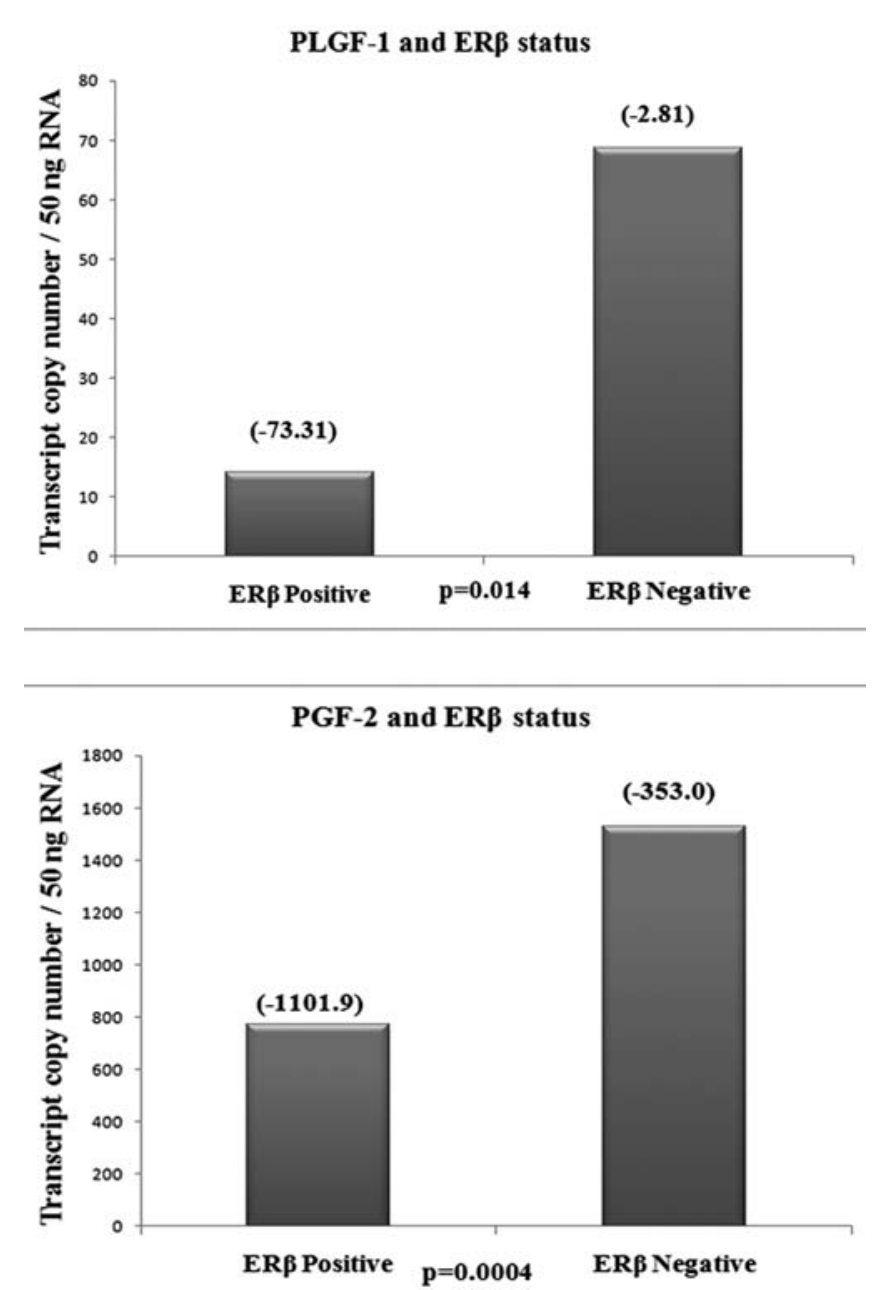

Figure 5. Levels of transcript of PLGF-1 and PGF-2 expression in nodepositive tumours compared with node-negative tumours.

for neuropilin-1 was also found to increase in conjunction with the increase in NPI indices, becoming significantly increased in patients with NPI $>5.4$ compared to with NPI $<3.4$. Our results for PLGF-1 and PGF-2 levels are increased in node-positive tumours, demonstrating the association of high levels of both isoforms with a poor prognosis in the patients.

The findings on neuropilin-1 are interesting, as it was only recently that this receptor was tested in clinical breast cancer. Similar to the findings in the present study, Ghosh et al (18) has shown that highly levels of neuropilin-1 is associated with a poor prognosis in patients with breast cancer. This, together with studies that peptides targeting certain regions of neuropilin results in the death of breast cancer cells which express neuropilin Barr (19), strongly indicate that targeting neuropilin-1 may have potential in new anti-cancer therapies. The correlation between PLGF-1, PGF-2 and neuropilin with ERß status is interesting. While PLGF-1 and PGF-2 were present at higher levels in ERßnegative tumours, neuropilin-1 results were the opposite. The mutual impact of PLGF-1/PGF-2 and ERß in breast is yet to be tested, but may prove to be an interesting lead to pursue, given the complex and uncertain nature of ERß in cell functions.
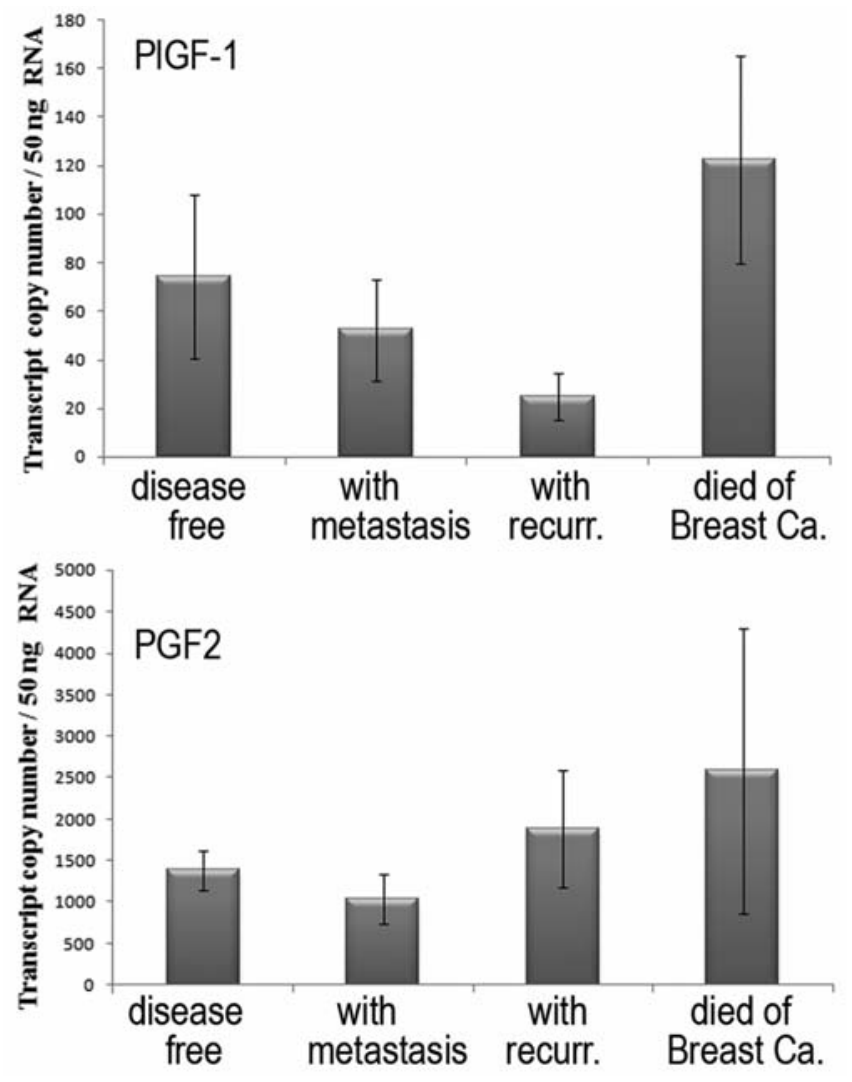

Figure 6. Comparison of transcript levels of PLGF-1 (top) and PGF-2 (bottom) in correlation with clinical outcome.

Table I. Results for neuropilin-1 expression in the patient cohort. ${ }^{\mathrm{a}}$

\begin{tabular}{lrrc}
\hline & \multicolumn{2}{c}{ Neuropilin-1 } & \\
\cline { 2 - 3 } Groups & Negative & Positive & P-value \\
(Fisher's test, two-tailed)
\end{tabular}

aData were obtained by scoring (high/low) RNA levels derived from Q-PCR data. The actual relative levels were too low for conventional analysis. 
In summary, this study has shown that PLGF-1 and PGF-2 expression of the angiogenic factor, $\mathrm{PGF}$, are increased in breast tumour tissues compared with normal breast tissues and together with the PGF receptor, neuropilin, display prognostic value in breast cancer patients. This indicates that blocking/ targeting PLGF-1 and PGF-2 expression may have a promising therapeutic future in breast cancer.

\section{Acknowledgements}

We wish to thank the ANGIOSTOP project (EU's Framework Programme 6) for funding this study. We also wish to thank Cancer Research Wales for continuing support of work in MARG.

\section{References}

1. Zetter BR: Angiogenesis and tumor metastasis. Annu Rev Med 49: 407-424, 1998.

2. Wirzenius M, Tammela T, Uutela M, et al: Distinct vascular endothelial growth factor signals for lymphatic vessel enlargement and sprouting. J Exp Med 204: 1431-1440, 2007.

3. Marcellini M, De Luca N, Riccioni T, et al: Increased melanoma growth and metastasis spreading in mice overexpressing placenta growth factor. Am J Pathol 169: 643-654, 2006.

4. Yang W, Ahn H, Hinrichs M, Torry RJ and Torry DS: Evidence of a novel isoform of placenta growth factor (PlGF-4) expressed in human trophoblast and endothelial cells. J Reprod Immunol 60: 53-60, 2003.

5. Roy H, Bhardwaj S and Yla-Herttuala S: Biology of vascular endothelial growth factors. FEBS Lett 580: 2879-2887, 2006.

6. Sawano A, Takahashi T, Yamaguchi S, Aonuma $\mathrm{M}$ and Shibuya M: Flt-1 but not KDR/Flk-1 tyrosine kinase is a receptor for placenta growth factor, which is related to vascular endothelial growth factor. Cell Growth Differ 7: 213-221, 1996.

7. Migdal M, Huppertz B, Tessler S, et al: Neuropilin-1 is a placenta growth factor-2 receptor. J Biol Chem 273: 22272 22278, 1998 .

8. Zhang L, Chen J, Ke Y, Mansel RE and Jiang WG: Expression of Placenta growth factor (PlGF) in non-small cell lung cancer (NSCLC) and the clinical and prognostic significance. World J Surg Oncol 3: 68, 2005.
9. Taylor AP and Goldenberg DM: Role of placenta growth factor in malignancy and evidence that an antagonistic PlGF/Flt-1 peptide inhibits the growth and metastasis of human breast cancer xenografts. Mol Cancer Ther 6: 524-531, 2007.

10. Staton CA, Kumar I, Reed MW and Brown NJ: Neuropilins in physiological and pathological angiogenesis. J Pathol 212: 237-248, 2007.

11. Parr C, Watkins G, Boulton M, Cai J and Jiang WG: Placenta growth factor is over-expressed and has prognostic value in human breast cancer. Eur J Cancer 41: 2819-2827, 2005.

12. Jiang WG, Douglas-Jones A and Mansel RE: Expression of peroxisome-proliferator activated receptor-gamma (PPARgamma) and the PPARgamma co-activator, PGC-1, in human breast cancer correlates with clinical outcomes. Int J Cancer 106: 752-757, 2003.

13. Jiang WG, Watkins G, Douglas-Jones A, Mokbel K, Mansel RE and Fodstad O: Expression of Com-1/P8 in human breast cancer and its relevance to clinical outcome and ER status. Int J Cancer 117: 730-737, 2005.

14. Martin TA, Parr C, Davies G, et al: Growth and angiogenesis of human breast cancer in a nude mouse tumour model is reduced by NK4, a HGF/SF antagonist. Carcinogenesis 24: 1317-1323, 2003.

15. Martin TA, Watkins G, Mansel RE and Jiang WG: Loss of tight junction plaque molecules in breast cancer tissues is associated with a poor prognosis in patients with breast cancer. Eur J Cancer 40: 2717-2725, 2004.

16. Parr C, Gan CH, Watkins G and Jiang WG: Reduced vascular endothelial growth inhibitor (VEGI) expression is associated with poor prognosis in breast cancer patients. Angiogenesis 9: 73-81, 2006.

17. Suen D and Chow LW: Prognostic contribution of the HER-2 oncogene overexpression to the Nottingham Prognostic Index in breast cancer. Biomed Pharmacother 60: 293-297, 2006.

18. Ghosh S, Sullivan CA, Zerkowski MP, et al: High levels of vascular endothelial growth factor and its receptors (VEGFR-1, VEGFR-2, neuropilin-1) are associated with worse outcome in breast cancer. Hum Pathol 39: 1835-1843, 2008.

19. Barr MP, Byrne AM, Duffy AM, et al: A peptide corresponding to the neuropilin-1-binding site on $\operatorname{VEGF}(165)$ induces apoptosis of neuropilin-1-expressing breast tumour cells. Br J Cancer 92: 328-333, 2005. 